Globe

Revue internationale d'études québécoises

\title{
De Versailles à Niamey. Le patrimoine constitutionnel canado-britannique du Québec et sa participation au sein de la Francophonie, 1968-1970 \\ From Versailles to Niamey. Québec's British-Canadian Constitutional Heritage and its Participation in the Francophonie, 1968-70
}

\section{David Meren}

Volume 13, numéro 1, 2010

Culture et relations internationales

URI : https://id.erudit.org/iderudit/044641ar

DOI : https://doi.org/10.7202/044641ar

\section{Aller au sommaire du numéro}

Éditeur(s)

Globe, Revue internationale d'études québécoises

ISSN

1481-5869 (imprimé)

1923-8231 (numérique)

Découvrir la revue

Citer cet article

Meren, D. (2010). De Versailles à Niamey. Le patrimoine constitutionnel canado-britannique du Québec et sa participation au sein de la Francophonie, 1968-1970. Globe, 13(1), 99-124. https://doi.org/10.7202/044641ar
Résumé de l'article

Cet article analyse les événements entourant le débat sur la nature de la participation du Québec au sein de la Francophonie, de l'affaire Gabon (1968) jusqu'à la deuxième conférence de Niamey (1970), afin d'explorer les origines et les inspirations de l'activité internationale du Québec. Les récits antérieurs ont mis l'accent sur l'intérêt manifesté par les néonationalistes pour le développement de relations avec la France et la communauté internationale francophone et sur l'idée que le Québec devait se projeter sur la scène internationale afin de protéger ses juridictions constitutionnelles contre les empiétements fédéraux. Cet article soutient que toute analyse des circonstances entourant la participation du Québec à l'institutionnalisation de la Francophonie et des efforts qu'il déploie pour affirmer sa « capacité internationale " doit aussi tenir compte de l'évolution parallèle du Canada vers la souveraineté internationale d'une part, et du fait que les actions posées par le Québec s'inspirent en partie de son patrimoine constitutionnel canado-britannique d'autre part. Pour le Québec, l'appui de la France s'avère certes crucial pour l'obtention et le maintien d'une place à la table de la Francophonie. Pourtant, en accord avec les éléments pragmatiques et gradualistes de sa culture politique -un legs de son patrimoine britannique le Québec a dû, tout au long de ce processus, faire preuve de prudence dans ses relations avec la France, tant il est vrai que la compréhension qu'a Paris des réalités et des enjeux québécois demeurait imparfaite. 


\title{
DE VERSAILLES À NIAMEY. LE PATRIMOINE CONSTITUTIONNEL CANADO-BRITANNIQUE DU QUÉBEC ET SA PARTICIPATION AU SEIN DE LA FRANCOPHONIE, $1968-1970$
}

\begin{abstract}
DAVID MEREN*
Université McGill Résumé - Cet article analyse les événements entourant le débat sur la nature de la participation du Québec au sein de la Francophonie, de l'affaire Gabon (1968) jusqu'à la deuxième conférence de Niamey (1970), afin d'explorer les origines et les inspirations de l'activité internationale du Québec. Les récits antérieurs ont mis l'accent sur l'intérêt manifesté par les néonationalistes pour le développement de relations avec la France et la communauté internationale francophone et sur l'idée que le Québec devait se projeter sur la scène internationale afin de protéger ses juridictions constitutionnelles contre les empiétements fédéraux. Cet article soutient que toute analyse des circonstances entourant la participation du Québec à l'institutionnalisation de la Francophonie et des efforts qu'il déploie pour affirmer sa «capacité internationale» doit aussi tenir compte de l'évolution parallèle du Canada vers la
\end{abstract}

$$
+\div
$$

\footnotetext{
* L'auteur tient à remercier le Conseil de recherches en sciences humaines du Canada pour son appui financier ainsi que les organisateurs du colloque Les Relations internationales du Québec dans tous leurs états. L'auteur remercie aussi Carman Miller pour son aide et ses encouragements, Christopher B. Hynes pour des clarifications sur des questions juridiques, de même que Jean-François Constant, Julie Allard et Nicolas Kenny qui ont aidé à traduire cet article.
} 
souveraineté internationale d'une part, et du fait que les actions posées par le Québec s'inspirent en partie de son patrimoine constitutionnel canado-britannique d'autre part. Pour le Québec, l'appui de la France s'avère certes crucial pour l'obtention et le maintien d'une place à la table de la Francophonie. Pourtant, en accord avec les éléments pragmatiques et gradualistes de sa culture politique - un legs de son patrimoine britannique -, le Québec a dû, tout au long de ce processus, faire preuve de prudence dans ses relations avec la France, tant il est vrai que la compréhension qu'a Paris des réalités et des enjeux québécois demeurait imparfaite.

\section{From Versailles to Niamey. Québec's British-Canadian Constitutional Heritage and its Participation in the Francophonie, 1968-70}

Abstract - This article examines the events regarding the debate over the nature of Québec's participation in the Francophonie from the Gabon Affair (1968) to the Second Niamey Conference (1970) in order to explore the origins of and inspirations for Québec's international activity. Previous accounts have emphasized the interest neo-nationalists had in cultivating ties with France and the international francophone community. Similarly prominent is the perceived need for Quebec to project itself internationally to protect against federal encroachments on its domestic jurisdiction. Building on such accounts, this article argues that discussion of the circumstances in which Quebec participated in the institutionalization of the Francophonie and affirmed its "international capacity" should be undertaken with an awareness of the parallels to Canada's evolution to international sovereignty, and the fact Quebec actions were inspired in part by its BritishCanadian constitutional heritage. To be sure, French assistance was crucial to Quebec obtaining and maintaining a distinct participation in the Francophonie and pursuing its international activities. Yet, consistent with the pragmatic and incrementalist political culture that derived from it's British heritage, Québec carefully managed its special relationship with a Paris that did not fully understand Québec realities.

Malgré des pourparlers ardus, ayant parfois soulevé la controverse, la France pouvait se vanter d'un succès diplomatique : l'organisme multilatéral qui venait de voir le jour présentait un véritable espoir pour une nouvelle ère de coopération internationale. L'une des controverses avait trait au statut de l'un des participants. Certains avaient exprimé des doutes quant à la personnalité internationale de ce dernier, un territoire manifestant un nationalisme croissant, étant donné son appartenance à une entité qui l'englobait et cherchait à maintenir au niveau international une unité de façade. Le participant en question réussit néanmoins à faire valoir la pertinence de sa présence, contribua de façon autonome aux discussions et put même signer le traité découlant de la conférence. C’est dans ces 
circonstances que le Canada participa à la conférence de Paris dans la foulée de la Première Guerre mondiale et signa le traité de Versailles (1919) pour ensuite devenir un membre de la Société des Nations. Considéré comme un moment fondateur d'une présence canadienne distincte au niveau international ${ }^{1}$, cet événement - du moins tel qu'il est présenté ici - rappelle étrangement les conditions de l'établissement d'une participation distincte du Québec au sein de l'Agence de coopération culturelle et technique (ACCT) et de la Francophonie.

La comparaison entre ces deux épisodes met en lumière à quel point les efforts déployés par le Québec sur la scène internationale, notamment pour l'obtention d'une participation distincte au sein de la Francophonie, s'inspirent de l'évolution canadienne vers la souveraineté internationale et de la transformation de l'Empire britannique en "Commonwealth". L'importance accordée par les historiens à la dimension française de l'épanouissement international du Québec fait en sorte que ce parallèle n'a pas été souligné jusqu'à maintenant. En effet, les événements liés aux retrouvailles franco-québécoises des années 1960, dont le point culminant demeure la visite de Charles de Gaulle, puis les tensions et les conflits opposant Québec, Ottawa et Paris par rapport à la participation du Québec à la Francophonie, donnent une allure très «franco-centrique " à l'historiographie ${ }^{2}$. Pourtant, une analyse des rapports triangulaires tendus depuis l'affaire Gabon de 1968 et jusqu'à la deuxième conférence de Niamey en 1970 révèle l'intérêt d'accorder une attention particulière aux sources d'inspiration du Québec dans ses efforts pour établir sa "capacité internationale " : l'interaction entre son patrimoine d'ancienne colonie britannique et son constitutionnalisme britannique, ainsi que l'émergence du Canada en tant qu'État souverain. L’analyse des documents de personnes clés impliquées dans les événements au Québec, notamment André Patry, Claude Morin et Jean Chapdelaine, révèle une approche fondamentalement pragmatique et

$$
\div+\div
$$

1. C.P. STACEY, Canada and the Age of Conflict. A History of Canadian External Policies, vol. 1 (18671921), Toronto, Macmillan of Canada, 1984, p. 240-258. Les autres dominions, à l'exception de TerreNeuve, ont aussi bénéficié d'une représentation distincte à la conférence de Paris.

2. Voir Robin GENDRON, Towards a Francophone Community. Canada's Relations with France and French Africa, 1945-1968, Montréal et Kingston, McGill-Queen's University Press, 2006; Stéphane PAQUIN, "La relation Québec-Paris-Ottawa et la création de l'Organisation internationale de la Francophonie (1960-2005) ", Guerres mondiales et conflits contemporains, vol. 54, nº 223, juillet 2006, p. 31-47 ; Frédéric BASTIEN, Relations particulières. La France face au Québec après de Gaulle, Montréal, Éditions du Boréal, 1999 ; J.L. Granatstein et Robert Bothwell, Pirouette. Pierre Trudeau and Canadian Foreign Policy, Toronto, University of Toronto Press, 1990 ; Dale Thomson, Vive le Québec libre!, Toronto, Deneau Press, 1988. 
gradualiste, attentive à l'accumulation et à la préservation de précédents ${ }^{3}$ qui contribueraient à l'établissement d'une coutume en droit susceptible de renforcer la capacité du Québec à contester l'interprétation fédérale de la constitution à propos de la responsabilité pour les affaires étrangères canadiennes. Ceci n'est pas sans rappeler la dynamique qui a marqué l'acquisition par le Canada d'une plus grande autonomie par rapport au Royaume-Uni et son accession à la souveraineté internationale. L'appui français apporté aux efforts québécois s'est avéré crucial à cet égard. Toutefois, le Québec a dû aussi gérer avec soin les relations particulières qu'il entretenait avec son allié français qui saisissait mal certaines nuances de la situation québécoise et qui avait évidemment ses propres intérêts à défendre. Donc, plutôt que de considérer le Québec comme le cœur d'une lutte entre Paris et Ottawa, ou comme l'acteur inférieur dans une relation bilatérale fédérale-provinciale ou franco-québécoise, on peut situer le Québec au sommet d'une dynamique triangulaire complexe. Dans cette dynamique, des acteurs québécois tiraient profit des occasions qui se présentaient pour augmenter l'envergure de l'action internationale du Québec.

Cet article commence par un survol du patrimoine britannique du Québec et propose un examen de la manière dont la population «canadienne" a adopté et adapté le constitutionnalisme britannique pendant l'ère coloniale, jusqu’à en faire une partie intégrante de sa culture politique. On verra comment les personnes impliquées dans ces efforts ont été influencées par la tradition britannique et se sont appropriées le legs anglais et l'exemple canadien afin de promouvoir leur propre cause. Suivra un examen de la lutte pour la reconnaissance de précédents pendant la Révolution tranquille, menée dans le contexte de la Francophonie naissante et culminant avec l'établissement de l'ACCT en 1970, alors que le Québec, assisté par Paris, cherchait à établir sa personnalité internationale tandis qu'Ottawa tâchait, pour sa part, de sauvegarder les prérogatives fédérales et de préserver l'unité internationale du Canada.

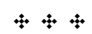

3. Dans cet article, j’emploie le mot "précédent " au sens conventionnel défini par Le Petit Robert: «un fait antérieur qui permet de comprendre un fait analogue : décision, manière d'agir dont on peut s'autoriser ensuite dans un cas semblable». D'ailleurs, "l'usage" est aussi invoqué comme source du droit international, c'est-à-dire que l'usage général des États peut être reconnu comme coutume et donc acceptée comme source du droit. À cet égard, voir J. Craig BARKER, International Law and International Relations. International relations for the $21^{\text {st }}$ century, New York, Continuum, 2000, p. 54-63. Cette acception de "précédent " differe de la définition classique légale du terme, reliée à la doctrine dans le droit commun anglais de stare decisis, le principe légal par lequel des points litigieux sont précisés en fonction de décisions antérieures. 


\section{QUÉBEC. UN PATRIMOINE «VERY BRITISH»}

Lutilisation par le Québec de son patrimoine juridique canado-britannique pour faire reconnaître sa capacité internationale dans les années 1960 s'inscrit dans une tradition bien établie au sein de la population francophone d'Amérique du Nord. Lawrence A.H. Smith a déjà décrit comment, au début du XIX siècle, les leaders "canadiens" se sont servis de la coutume et de la jurisprudence constitutionnelle britannique dans leurs luttes politiques contre le gouverneur James Craig et la minorité Tory et britannique qui souhaitaient priver la population canadienne-française d'une représentation politique adéquate tout en poursuivant un objectif d'assimilation. C'est ainsi que la volonté de préserver le caractère "canadien " de l'assemblée législative du Bas-Canada s'est accompagnée d'un désir de rendre celle-ci institutionnellement plus "britannique " ${ }^{4}$. Yvan Lamonde va dans le même sens lorsqu'il avance qu'en 1814, les Canadiens lancèrent un appel à Londres pour obtenir une forme de responsabilité ministérielle en prétextant qu'ils " [n'avaient] point d'autres ressources que dans la protection de la mèrepatrie» pour défendre leurs intérêts. Cet appel se solda par un échec, mais ce scénario se répéta quinze ans plus tard lorsqu'il s'est agi de réclamer l'élection du Conseil législatif - jusque-là nommé par le gouverneur - dans l'espoir d'accroître l'influence canadienne au niveau parlementaire. Comme le remarque Lamonde, la dimension ethnique des luttes politiques de la colonie ne s'est pas traduite, du moins jusqu'aux années 1830, par un rejet de la tradition et des institutions britanniques. Au contraire, les dirigeants canadiens en profitèrent pour assurer la survie de la société canadienne. Ces efforts, tels ceux de Denis-Benjamin Viger, envoyé à Londres en qualité de représentant du Bas-Canada malgré l'objection du Conseil législatif, préfigurent en quelque sorte l'activité internationale du Québec 5 .

Lamonde identifie trois traditions ayant influencé les relations $\mathrm{du}$ Bas-Canada avec le Royaume-Uni. Il relève, dans un premier temps, un loyalisme clérical qui ne remet pas en cause le nouveau régime britannique. Une deuxième approche, la loyauté conditionnelle, qui s'accorde davantage avec la tradition politique libérale, est marquée par une critique des autorités coloniales et britanniques. Elle aboutit ultimement à la rupture des années 1830. C'est la troisième tradition, toutefois, celle d'un pragmatisme

$$
\div+\div
$$

4. Lawrence A.H. SMITH, "Le Canadien and the British Constitution, 1806-1810", Canadian Historical Review, vol. 38, no 2, juin 1957, p. 97-101.

5. Yvan LAMONDE, Allégeances et dépendances. L'histoire d'une ambivalence identitaire, Québec, Éditions Nota bene, 2001, p. 171-173. 
conservateur caractérisé par une volonté de travailler à l'intérieur du cadre colonial afin d'obtenir le plus d'avantages possible, qui rejoint le mieux le propos de cet article. Cette approche pragmatique, incarnée, entre autres, par Louis-Hippolyte La Fontaine et George-Étienne Cartier, a perduré au-delà des années 1830, et est devenue l'élément dominant de la culture politique du Canada-Est sous le régime mis en place après l'Acte d'Union (1841). La collaboration avec des politiciens du Canada-Ouest a d'abord mené à l'établissement du gouvernement responsable, puis, à la faveur de la Confédération (1867), au rétablissement, au Québec, d'une assemblée législative à majorité francophone au sein du système fédéral. Au cours des années subséquentes, Wilfrid Laurier s'est fait l'héritier de cette tradition, louant, dans son fameux discours sur le libéralisme politique prononcé devant le Club canadien en 1877 qui en fit une personnalité politique importante sur la scène nationale, le fait que les Canadiens français se soient servis des institutions politiques et constitutionnelles britanniques pour assurer leur survie $^{6}$. Même avec l'affaiblissement du lien britannique, cette ligne de conduite conservatrice et pragmatique s'est perpétuée et influence encore les mouvements nationalistes et souverainistes, notamment la stratégie étapiste du Parti Québécois ${ }^{7}$.

Cette approche rappelle le caractère gradualiste de la tradition politique et légale britannique et contraste, par exemple, avec les aspects rationalistes et révolutionnaires de la culture politique française. Il est décelable dans la nature organique et évolutionniste de la constitution du Royaume-Uni. Le pragmatisme est encore au cour de la tradition du droit commun anglais, un système judiciaire basé sur les "précédents » créés par les décisions antérieures des tribunaux, sur les doctrines motivant implicitement ces décisions de même que sur les coutumes et les usages, plutôt que sur des lois écrites et codifiées. Le concept du stare decisis, qui émerge comme concept légal fort au XIX ${ }^{\mathrm{e}}$ siècle et s'appuie sur une tradition politique et légale du précédent plusieurs fois centenaire, est crucial à cet égard. Tout

$$
+\div
$$

6. Ibid., p. 180-183 et 193. Voir aussi Jocelyn LÉTOURnEAU, A History for the Future: Rewriting Memory and Identity in Quebec, Montréal, McGill-Queen's University Press, 2004, p. 128-131. Létourneau recommande de sortir du récit d'adversité qui caractérise souvent l'histoire du Québec afin de reconnaître l'habileté avec laquelle les francophones du Québec ont tiré profit des occasions politiques qui se sont présentées à eux. Il insiste également sur l'importance de situer l'expérience du Québec dans les histoires plus larges du monde atlantique, de l'empire britannique et du Canada.

7. Voir Jean CHrÉTIEN, Dans la fosse aux lions, Montréal, Éditions de l'Homme, 1994, p. 140. Morin aurait dit à Jean Chrétien : "Nous nous séparerons du Canada de la même manière que le Canada s'est séparé de l'Angleterre. Nous couperons les liens un par un, nous obtiendrons une petite concession ici, une petite concession là et, finalement, il ne restera plus rien". 
aussi important, le concept de convention constitutionnelle, c'est-à-dire les coutumes, les pratiques, les maximes et les préceptes qui, bien que ne formant pas un corps de lois per se, constituent néanmoins un ensemble cohérent de règles éthiques et politiques, contribue également à la définition du constitutionnalisme canado-britannique ${ }^{8}$.

Les Canadiens français ont joué un rôle central dans l'évolution lente et parfois pénible du Canada vers la souveraineté internationale. En 1882, le Québec délégua Hector Fabre à Paris comme agent général. Peu de temps après, le même Fabre fut nommé par Ottawa au poste de Commissaire dans la capitale française, un premier pas vers une représentation canadienne officielle à l'extérieur de l'Empire britannique. En 1923, Ernest Lapointe, à l'époque ministre fédéral de la Marine et des Pêcheries, incita le premier ministre Mackenzie King à signer, indépendamment de Londres, un traité de pêche projeté avec les États-Unis. L'importance du Traité du flétan (1923) dépassait largement la simple question des pêches dans le Pacifique Nord : en signant le traité sans la participation des autorités britanniques, Lapointe créait un précédent capital qui contribua à l'acquisition pour le Canada d'une nouvelle compétence en matière de négociation de traités internationaux. Plus tard, alors qu'il occupait le poste de ministre de la Justice, Lapointe présida la délégation canadienne lors des discussions qui menèrent au Statut de Westminster (1931) qui enchâssait la reconnaissance de la souveraineté internationale du Canada, un fait officieusement reconnu depuis la déclaration Balfour de $1926^{9}$.

En 1961, à l'occasion de l'inauguration de la Maison du Québec à Paris, le premier ministre Jean Lesage ne manqua d'ailleurs pas de souligner que les Canadiens français s'étaient appropriés les institutions politiques

$$
\div+\div
$$

8. Sur la constitution et le constitutionnalisme britannique, voir J.W. F. ALLISON, The English Historical Constitution. Continuity, Change and European Effects, Cambridge, Cambridge University Press, 2007. Le concept légal de "précédent" et la tradition du droit commun anglais sont examinés par Stephen WADDAMS, "Authority, Precedent, and Principle», University of Toronto Law Journal, vol. 59, $\mathrm{n}^{\circ} 1$, 2009, p. 127-133 : Rupert Cross and J.W. HARRIS, Precedent in English Law, $4^{\mathrm{e}}$ édition, Oxford, Clarendon Press, 1991. Le concept de convention constitutionnelle a été étudié par Albert Venn DICEY, An Introduction to the Study of the Law of the Constitution, London, Macmillan, 1959, p. 417 et 469-470. La théorie du droit commun constitutionnel est également pertinente dans le contexte canadien. Issue de la tradition du droit commun anglais, cette théorie veut que les tribunaux peuvent, et même doivent, enrichir la signification originale de textes constitutionnels généraux ou ambigus, en se référant à la tradition et au précédent. Pour une discussion de ce concept, central dans la tradition légale américaine, voir Adrian Vermeule, "Common Law Constitutionalism and the Limits of Reason", Columbia Law Review, vol. $107, \mathrm{n}^{\circ} 6,2007$, p. 1482-1532.

9. John Hilliker, Canada's Department of External Affairs. The Early Years. 1909-1946, Montréal et Kingston, McGill-Queen's University Press, 1990, p. 17 et 92. Londres autorise alors une signature canadienne indépendante de la sienne, un geste considéré comme le moindre de deux maux après que King eut menacé d'ouvrir une légation à Washington le cas échéant. 
britanniques afin d'assurer la survivance et le développement du fait français en Amérique ${ }^{10}$. De leur côté, les responsables français remarquèrent le caractère "britannique » du Québec. L'ambassadeur François Leduc, par exemple, dans un rapport au sujet de l'avenir politique du Canada qu'il envoya à Paris au début de 1967, affirma que le Canada lui paraissait «voué à s'adapter petit à petit, avec un pragmatisme bien britannique dont les Québécois [étaient] eux-mêmes imprégnés, à une nouvelle forme de cohabitation $^{11}$ ».

De nombreuses personnalités impliquées dans l'avancement de la capacité internationale du Québec ont été influencées par la tradition juridique et constitutionnelle britannique, car ils avaient évolué dans ce qu'il convient d'appeler un milieu intellectuel «britannique». Paul Gérin-Lajoie, ministre de l'Éducation et vice-premier ministre dans le gouvernement Lesage, était un expert de l'évolution constitutionnelle du Canada, de son statut de colonie britannique jusqu'à sa souveraineté. Il avait obtenu son doctorat à l'université Oxford en tant que boursier Rhodes. Jean Chapdelaine, le délégué général du Québec à Paris de 1965 à 1976, avait aussi poursuivi des études à Oxford en bénéficiant d'une bourse Rhodes. Louis Bernard qui, dans les années 1960, était conseiller juridique au ministère provincial des Affaires fédérales-provinciales a rédigé à l'Université de Londres une thèse en droit sur la compétence provinciale de négocier et de conclure des accords internationaux ${ }^{12}$. Jacques-Yvan Morin, à l'époque professeur de droit international et constitutionnel à l'Université de Montréal et conseiller des gouvernements Lesage et Johnson, avait acquis une connaissance approfondie de la tradition constitutionnelle britannique grâce à ses études en droit aux universités McGill et Cambridge. Un autre conseiller du gouvernement, Jacques Brossard, a fait ses études au Balliol College (Oxford) et a servi en tant qu'agent au service extérieur canadien avant son retour au Québec pour enseigner le droit à l'Université de Montréal. Claude Morin, sous-ministre des Affaires fédérales-provinciales et André Patry, conseiller au gouvernement Lesage et nommé chef du protocole par Daniel Johnson, avaient fait leurs études au Québec, ce qui ne les a pas empêchés d'adopter

$$
+\div
$$

10. Dale Thomson, Vive le Québec libre!, op cit., p. 98.

11. Ministère des Affaires étrangères, Série B, Amérique, Sous-série Canada, 1964-1970 [MAE], vol. 265 - Télégramme de Leduc au MAE, Amérique, 4 janvier 1967 ; MAE, vol. 198 - Télégramme de Leduc au MAE, Amérique, 8 avril 1965. Deux ans plus tôt, Leduc écrivait que Lesage restait, sans même s'en rendre compte, imprégné d'habitudes anglaises, nonobstant sa volonté affichée d'incarner la pensée et la culture françaises.

12. Dale THOMSON, op cit., p. 127. 
l'approche pragmatique, qui s'accordait avec la tradition constitutionnelle canado-britannique, dans leurs efforts pour réclamer une capacité internationale pour le Québec. En présentant leurs arguments en faveur d'une telle capacité, ces dirigeants s'inspiraient certainement de leur formation universitaire et de leur connaissance du droit international. Ils mettaient l'accent sur la coutume et la jurisprudence, ce qui constituait une voie quelque peu analogue à celle empruntée dans le common law anglais. Leurs initiatives furent aussi influencées par les traditions constitutionnelles et juridiques britanniques auxquelles ils avaient été exposés tant au cours de leurs études que durant leur vie professionnelle.

\section{DE LA COLONIE À LA NATION. LES ARGUMENTS EN FAVEUR D'UNE PERSONNALITÉ INTERNATIONALE POUR LE QUÉBEC}

Ces personnes participèrent à la formulation et à la promotion de la doctrine qui soutient l'action internationale du Québec et à laquelle Paul GérinLajoie a donné son nom. L'origine de cette doctrine peut être identifiée dans l'histoire constitutionnelle du Canada et dans les ambiguïtés de la compétence canadienne en matière d'affaires étrangères. L'Acte de l'Amérique du Nord britannique (AANB) de 1867 qui établit le Dominion du Canada a été conçu à l'origine comme un outil pour faciliter l'autonomie interne du Dominion. Il tenait pour acquis que la nouvelle entité politique demeurerait une colonie subordonnée au Royaume-Uni au niveau international. Avec l'émergence du Canada comme acteur autonome sur la scène internationale, la question de la responsabilité constitutionnelle en affaires étrangères devint un enjeu important puisque, bien que l'AANB instituait une union fédérale, il ne conférait aucune compétence en affaires étrangères, sauf à la section 132 qui prévoyait que le gouvernement central mettrait en œuvre les traités conclus par l'Empire britannique (c.-à-d. Londres). Selon Ottawa, le pouvoir résiduel dans la section 91 de l'AANB conférait au fédéral une compétence exclusive en matière d'affaires étrangères. Au cours des années 1930, les provinces, motivées par la crainte des empiétements d'Ottawa sur ce qui relevait de la juridiction provinciale, contestèrent cette compétence fédérale. L'affaire des Conventions du travail (1937) aboutit au Comité judiciaire du Conseil privé $(\mathrm{CJCP})$ et mit fin à cette exclusivité fédérale en rendant une décision qui limitait la capacité d'Ottawa à mettre en œuvre les traités internationaux aux seuls champs relevant de la compétence fédérale. Toutefois, la décision du CJCP restait muette sur la question de la 
compétence - fédérale ou provinciale - de négocier et de conclure des traités internationaux ${ }^{13}$.

Ottawa refusait de reconnaître un jus tractatuum des provinces, employant l'argument selon lequel seul le gouvernement fédéral avait le droit de traiter avec les puissances étrangères, y compris dans les domaines qui relevaient de compétences provinciales ${ }^{14}$. La sensibilité fédérale à ce propos était exacerbée par l'accroissement des échanges transnationaux et l'interdépendance économique des États poussait les relations internationales vers des sujets techniques relevant traditionnellement de la politique intérieure et donc de la compétence constitutionnelle des provinces ${ }^{15}$. Pour Québec, au contraire, la logique prescrivait qu'un environnement de plus en plus mondialisé nécessitait l'extension de ses juridictions à l'étranger ${ }^{16}$.

On trouve la présentation la plus claire de la position québécoise dans le discours donné par Gérin-Lajoie en avril 1965 aux corps consulaires de Montréal. Il affirma alors que le Québec possédait une capacité internationale en vertu de sa souveraineté dans ses domaines de juridiction. Le vice-premier ministre soutenait qu'à la lumière de l'affaire des Conventions $d u$ travail, qui limitait la capacité fédérale de mettre en œuvre des traités touchant à la juridiction provinciale, il était fondé de postuler que la compétence des provinces dans les domaines de juridiction provinciale ne se limitait pas à l'entérinement et à l'exécution des traités, mais comportait également une capacité de négocier et de conclure des conventions internationales. Gérin-Lajoie considérait ce fait d'autant plus important que les relations internationales concernaient de plus en plus des matières relevant de la compétence provinciale. En plus de ce jus tractatuum restreint, il en appelait à une participation distincte du Québec dans les organisations internationales qui œuvraient dans des domaines relevant de la compétence provinciale ${ }^{17}$.

$$
\div+\div
$$

13. Howard A. LEESON et Wilfrid V. VANDERELST, External Affairs and Canadian Federalism. The History of a Dilemma, Holt, Rinehart and Winston of Canada Ltd., 1973, p. 2, 62 et 88; Anne-Marie JACOMYMileTTE, "Aspects juridiques des activités internationales du Québec », Paul PAINCHAUD (dir.), Le Canada et le Québec sur la scène internationale, Québec, Presses de l'Université du Québec, 1977, p. 515-544.

14. Département des Affaires extérieures [DAE], G-2, vol. 3197, 5175-40 - Mémorandum de la Division européenne au SSEAE, 31 mai 1960 ; DAE, G-2, vol. 4286 et, 10605-A-40 - Mémoire de T.H.W. Read, 11 mai 1955, Provincial Representation Abroad.

15. Renaud DEHOUSSE, «Fédéralisme, asymétrie et interdépendance. Aux origines de l'action internationale des composantes de l'État fédéral ", Études Internationales, vol. 20, no 2, 1989, p. 284-288.

16. Louis BALTHAZAR, "Quebec's International Relations. A Response to Needs and Necessities », Brian HoCKING (dir.), Foreign Relations and Federal States, New York, Leicester University Press, 1993, p. 143145 .

17. Bibliothèque et archives nationales Québec [BAnQ], P422, S2, 1995-01-008, vol. 2 et 5 - Allocution du ministre de l'Éducation, Monsieur Paul Gérin-Lajoie, aux membres du Corps consulaire de Montréal, 12 avril 1965. Voir aussi, Jacques BROSSARD, André PATRY et Élisabeth WEISER, Les pouvoirs extérieurs du Québec, Montréal, Les Presses de l’Université de Montréal, 1967. 
Dans les jours suivant l'énoncé de la doctrine Gérin-Lajoie concernant le prolongement externe des compétences internes du Québec, Claude Morin employa l'approche pragmatique qui s'accordait avec la tradition constitutionnelle canado-britannique. Il avoua au premier ministre Lesage qu'il n'était pas certain que le Québec puisse justifier le bien-fondé juridique de la doctrine Gérin-Lajoie, mais qu'une solution politique était nécessaire puisque le statu quo concernant la compétence canadienne en politique étrangère était devenu inacceptable ${ }^{18}$. Le premier ministre canadien Lester Pearson était prêt à être conciliant mais Marcel Cadieux, soussecrétaire d'État aux Affaires extérieures, l'avertit que la démarche était inconstitutionnelle et diminuerait la personnalité internationale du Canada ${ }^{19}$. Déterminé à vaincre le défi néonationaliste posé à la prérogative fédérale, Cadieux soutenait l'idée qu'il fallait canaliser l'activité internationale du Québec dans une politique étrangère canadienne plus large qui réfléchirait mieux la réalité biculturelle du pays. Il s'opposait cependant résolument à la suggestion selon laquelle le Québec pourrait jouer un rôle «national» sur la scène internationale ${ }^{20}$.

La volonté d'affirmation internationale du Québec s'étendait évidemment à la Francophonie, un forum crucial qui permettait au Québec de poursuivre sa vocation culturelle et de mettre en avant la doctrine GérinLajoie. À l'été 1966, Jean Chapdelaine favorisa une Francophonie plus pragmatique, souple, et non gouvernementale; il s'inquiétait du fait que les efforts déployés pour institutionnaliser les liens francophones puissent entraîner l'implication d'Ottawa comme interlocuteur. Chapdelaine soutenait que le réseau de l'Association des universités partiellement ou entièrement de langue française suffisait aux ministres, aux parlementaires et aux fonctionnaires québécois pour maintenir le contact avec leurs homologues d'outre-mer. La multiplication de tels rapports bilatéraux, toujours selon Chapdelaine, constituait le maximum que le Québec pouvait espérer dans la conjoncture constitutionnelle du moment. Claude Morin était d'accord, et affirmait à cette époque que si l'encouragement à la coopération francophone était une bonne chose, il convenait d'éviter que celui-ci se traduise par une concurrence avec Ottawa et que le fédéral soit tenté

$$
4+4
$$

18. BAnQ, P762, 1999-10-011, vol. 60, Mémoires aux premiers ministres/Mémos de M. Morin aux premiers ministres, 1962-1976 - Mémoire de Morin à Lesage, 30 avril 1965, voyage à Ottawa.

19. Thomson (1988), 156-157: BAnQ, E5, 1986-03-007, vol. 94, p. 1, 1965 - Mémoire des délibérations du Conseil exécutif, Séance du 11 mai 1965.

20. DAE, A-3-c, vol. 10141, 30-12-QUE, p. 3 - Memorandum from Cadieux to the SSEA, 4 February 1966, The Provinces and International Relations, refers to a memo from SSEA, 17 December 1965. 
d'institutionnaliser la Francophonie afin d'asseoir sa prérogative en affaires étrangères ${ }^{21}$.

En effet, les fonctionnaires fédéraux considérèrent initialement l'institutionnalisation de la Francophonie comme un avantage puisqu'elle renforcerait la position fédérale en affaires étrangères. Des craintes furent exprimées au sein du département des Affaires extérieures selon lesquelles l'inaction d'Ottawa pourrait inciter ceux qui travaillaient, au Québec et à l'étranger, à l'établissement d'une personnalité distincte du Québec à passer à l'action ${ }^{22}$. La question de la participation du Québec dans la Francophonie devint plus urgente après le «cri du balcon» du Général de Gaulle. Le père Georges-Henri Lévesque, à l'époque recteur de l'Université nationale du Rwanda, sonna l'alarme à Ottawa après la réunion fondatrice de l'Association des universités africaines au Maroc, au cours de laquelle l'indépendantiste Jean-Marc Léger promut l'idée d'une participation directe du Québec dans la Francophonie. Lévesque pressa Ottawa de nommer un diplomate de grande envergure afin de neutraliser les efforts de Léger qui pourrait " connaître son Austerlitz si on ne lui prépare pas un Trafalgar ${ }^{23}$ ".

\section{UN COUP DE POUCE FRANÇAIS}

Le débat sur la participation du Québec dans la Francophonie permet d'éclaircir le rôle déterminant joué par la France dans l'action internationale du Québec et sa rivalité avec Ottawa au sujet de la compétence en affaires étrangères. Les parallèles entre le droit international et le droit commun anglais favorisaient la coopération des nationalismes québécois et gaulliste. Louis Bernard conseilla au gouvernement Lesage de profiter de ses relations avec Paris pour convaincre les dirigeants français de signer un accord intergouvernemental avec le Québec, établissant du même coup un précédent en ce qui concerne d'abord la compétence du Québec à négocier et à conclure

$$
+\div
$$

21. BAnQ, P422, S2, 1995-01-008, vol. 3 et 5, Lettre de Chapdelaine à Morin, 11 juillet 1966, francophonie; BAnQ, E42, 1990-09-002, vol. 405, Délégation du Québec à Paris, 1966-1967, B Lettre de Morin à Chapdelaine, 26 juillet 1966.

22. DAE, A-3-c, vol. 11632, 30-10-FRAN, p. 1 - Visit of President Senghor, September 19-21, "La Francophonie », 13 septembre 1966; DAE, A-3-c, vol. 10685, 26-2-CDA-QUE, p. 1 - Mémorandum de Gotlieb, Services juridiques, à la Division européenne, 27 septembre 1966, Constitutional Implications of Quebec's Participation in the Proposed French Community; DAE, A-3-c, vol. 10684, 20-6CDA, p. 1 - Mémorandum de Halstead, Division européenne, au SSEAE, 24 octobre 1966, Le Canada et la Francophonie.

23. DAE, A-3-c, vol. 11632, 30-10-FRAN, p. 1 - Mémorandum de Beesley, Services juridiques, à Cadieux, 14 décembre 1967, Fondation au Maroc de l'Association des universités africaines : rôles du Père G.H. Lévesque et de Jean-Marc Léger. 
des traités, puis le prolongement international de sa juridiction constitutionnelle, qu'il pourrait ensuite soumettre à la Cour Suprême du Canada ${ }^{24}$. Le rapprochement officiel franco-québécois des années soixante, rendu manifeste par les ententes en matière d'éducation et de coopération culturelle (1965), la visite du Général de Gaulle (1967) et par les accords de coopération Johnson-Peyrefitte (1967), joua un rôle crucial dans la concurrence entre Québec et Ottawa. Les autorités fédérales se retrouvaient ainsi dans une position qui rappelait celle de Londres pendant les premières décennies $\mathrm{du} \mathrm{XX}^{\mathrm{e}}$ siècle. Soucieuse de maintenir l'unité internationale de l'Empire britannique, Londres s'était alors méfiée de tous les efforts des dominions, y compris ceux du Canada, pour affirmer une personnalité internationale séparée. Pendant la Révolution tranquille, le Québec était dans la même situation que le Dominion du Canada au cours des années qui avaient précédé la déclaration Balfour et le Statut de Westminster, puisqu'il revendiquait de plus en plus une personnalité internationale indépendante. La tendance qu'avait Paris à traiter directement avec Québec représentait un appui implicite (et de plus en plus explicite) à la doctrine Gérin-Lajoie. En effet, de Gaulle envisageait l'aide de la France dans l'établissement d'une personnalité internationale pour le Québec comme une étape vers la réalisation de son objectif politique plus large : favoriser l'indépendance du Québec ${ }^{25}$. Même avant le "cri du balcon", Morin constatait que, bien que l'accroissement de l'activité internationale du Québec n'avait pas provoqué de modifications constitutionnelles au sens juridique, des gestes concrets, comme l'ouverture de la Délégation générale du Québec à Paris, la signature d'ententes avec la France (sanctionnées cependant par un échange de lettres entre les autorités fédérales et françaises), et la prolifération des missions gouvernementales québécoises à l'étranger, transformaient de facto la dynamique constitutionnelle: le Québec était plus que jamais actif sur la

$$
\div+\div
$$

24. Dale ThOMSON, op cit., p. 127.

25. Alain PeYrefiTte, De Gaulle et le Québec, Montréal, Stanké, 2000, p. 88-90. Peyrefitte cite de Gaulle qui aurait dit explicitement: "nous allons proposer au Québec une coopération bilatérale de plus en plus étroite, qui aboutira de facto à ce que la France traite le Québec comme un État souverain. " Par la suite, De Gaulle a refusé d'assister à une réception tenue par l'Association internationale des parlementaires de langue française lors de sa réunion de septembre 1968, à laquelle il semblait que le Québec n'assisterait pas, puisqu'il s'opposait au fait que le Québec n'ait pas droit à une adhésion séparée au sein de l'organisation. De Gaulle a alors déclaré: "Je n’irai pas s'il n’y a pas le Québec, ce serait ridicule ! C’est complètement grotesque!». Ce n'est que sur l'insistance d'un proche conseiller, Jacques Foccart, déterminé à le persuader d'y assister, qu'il a finalement décidé de s'y rendre. Jacques FocCART, Journal de l'Elysée, tome 2. Le Général en mai (1968-1969), Paris, Fayard/Jeune Afrique, 1998, p. 343-345. 
scène internationale ${ }^{26}$. Ottawa était tout à fait conscient de l'exactitude de l'analyse de Morin ; l'annonce de l'établissement du ministère des Affaires intergouvernementales (MAIQ) à la fin du mois de février 1967 provoqua d'ailleurs une vive réaction de la part du gouvernement fédéral. Conscient des parallèles historiques qui pouvaient être établis avec la création du département des Affaires extérieures et du rôle que celui-ci avait joué dans l'affirmation par le Canada d'une personnalité internationale distincte et son indépendance du Royaume-Uni, Pearson déclara qu'Ottawa s'opposerait résolument à tous les efforts du Québec pour employer le MAIQ comme outil pour signer des accords internationaux de manière indépendante ${ }^{27}$. C'est d'ailleurs plus ou moins ce que Jean Chapdelaine soutenait dans une analyse de la réponse fédérale à l'institutionnalisation des relations francoquébécoises, qui s'appliquait aussi à la question de la participation du Québec au sein de la Francophonie: l'appui français permettrait au Québec de placer Ottawa devant des faits accomplis, laissant aux juristes le soin des détails ${ }^{28}$.

L'un de ces faits accomplis concerne l'invitation du Québec, après une intercession française auprès des autorités gabonaises, à la conférence des ministres francophones de l'Éducation à Libreville en février 1968, organisée par l'Organisation commune africaine et malgache. Encouragé par ses diplomates, politiciens et hauts fonctionnaires membres du «lobby québécois » à Paris, de Gaulle perçut ce geste comme s'accordant avec son ambition d'établir une personnalité internationale distincte pour le Québec ${ }^{29}$. À Libreville, le ministre français de l'Éducation nationale, Alain Peyrefitte, profita de ses discours publics pour attirer l'attention sur la participation du Québec à la conférence à titre de membre participant de la Francophonie, et non pas en qualité d'observateur, soulignant par le fait même l'entrée du Québec sur la scène internationale ${ }^{30}$.

$$
\div+\div
$$

26. Claude MORIN, L'Art de l'impossible. La diplomatie québécoise depuis 1960, Montréal, Les Éditions du Boréal Express, 1987, p. 61.

27. MAE, vol. 278 - Telegram from Leduc to MAE, Amérique, 2 March 1967.

28. BAnQ, P776, 2001-01-006, vol. 2, Daniel Johnson, 1966-1969, Lettre de Chapdelaine à Johnson, 30 novembre 1967.

29. BAnQ, P776, 2001-01-006, vol. 4, Ministère des Affaires Intergouvernementales, 1969-1996 Lettre de Dorin à Masse, 25 mars 1996; BAnQ, P776, 2001-01-006, vol. 4, Divers - Lettre de Peyrefitte à Chapdelaine, 6 juillet 1998 ; Alain PEYREFITTE, op. cit., p. 85 ; Jacques FOCCART, op cit., p. 343-345. 30. Robin GENDRON, op cit., p. 133. 


\section{LA LUTTE POUR CRÉER DES PRÉCÉDENTS : DE LIBREVILLE À NIAMEY}

Québec afficha sa volonté d'accumuler les précédents soutenant une plus grande autonomie au niveau international, et ce, dès le début de «l'affaire Gabon ». Convaincu que le Québec devait être invité directement à participer à la réunion de Libreville, Daniel Johnson s'opposa à toute ingérence fédérale arguant que la réunion portait sur l'éducation ${ }^{31}$. Il évita donc de répondre aux propositions fédérales concernant la participation du Québec au sein d'une délégation canadienne, notamment à celle qui voulait que le ministre de l'Éducation du Québec, Jean-Guy Cardinal, en soit le président. Cardinal se rendit plutôt à Libreville avec un mandat clair de la part de Johnson : «Allez-y fort. Je vous envoie [...] pour faire l'agent provocateur»! Cardinal était d'ailleurs porteur d'une lettre de créance attestant qu'il représentait le gouvernement du Québec en son nom propre ${ }^{32}$.

La volonté d'accumuler des précédents pour renforcer la position du Québec dans ses pourparlers avec Ottawa au sujet de la compétence en matière d'affaires étrangères transparaît également dans la façon dont les dirigeants québécois utilisèrent l'aide française. Par exemple, à la fin de décembre 1967, Claude Morin fit remarquer à l'ambassadeur Leduc que Québec souhaitait recevoir une invitation directe à Libreville, donc sans le truchement d'Ottawa. Il assura au diplomate que Québec s'occuperait des difficultés avec le gouvernement fédéral qui pourraient en découler ${ }^{33}$. Chapdelaine poursuivit le travail de Morin à Paris et précisa que le Québec n'assisterait pas à la conférence sans avoir reçu une invitation directe. Dans leurs démarches, les deux responsables québécois rejetèrent les mesures envisagées pour apaiser Ottawa et prescrites par certains éléments plus prudents du Quai d'Orsay, une prudence qui contrastait avec l'indifférence relative manifestée par l'Élysée à l'égard de la réaction fédérale ${ }^{34}$.

L'affaire Gabon et ses suites offrent également un exemple des efforts déployés par le Québec afin de profiter de l'aide française tout en évitant une crise importante avec Ottawa. Bien que Daniel Johnson ait cru que le Québec devait participer seul à Libreville, il donna aussi des instructions à

$$
4+4
$$

31. Claude MORIN, op. cit., p. 116.

32. Ibid., p. 117-122; Pierre GODIN, Daniel Johnson 1964-1968, la difficile recherche de l'égalité, Montréal, Les Éditions de l'Homme, 1980, p. 316.

33. BAnQ, E42, 1995-02-001, vol. 147, CME, Statut du Québec, Général-Mémoire de Morin à Johnson, 22 décembre 1967.

34. Frédéric BASTIEN, op. cit., p. 51 ; Alain PeYRefiTTe, op. cit., p. 110; BAnQ, P776, 2001-01-006, vol. 3, Dossier Personnel - Lettre de Chapdelaine à Morin, 11 janvier 1968 ; Robin GENDRON, op. cit., p. 132. 
Morin afin qu'il évite les «histoires inutiles» avec le fédéral. Lorsque le Québec reçut l'invitation officielle pour participer à la conférence de Libreville à la mi-janvier 1968, Morin choisit de ne pas profiter de l'occasion pour parvenir à un accord durable avec Ottawa garantissant une participation distincte du Québec aux réunions internationales qui toucheraient à des domaines de compétence provinciale. Selon lui, cette décision s'explique, d'une part, par la crainte qu'une entente avec le gouvernement fédéral soit interprétée par les alliés de Québec (Paris) comme un recul et une trahison et, d'autre part, par l'impression que d'autres invitations semblables allaient suivre celle de Libreville, ce qui renforcerait d'autant plus la position du Québec vis-à-vis d'Ottawa ${ }^{35}$. Pourtant, un an après l'affaire Gabon, Morin reconnaissait que la position du Québec était « dangereusement avancée » à la suite des circonstances de sa participation à la réunion de Libreville et de la réaction fédérale que cette participation avait provoquée ${ }^{36}$.

Au sujet du deuxième volet de la conférence de Libreville tenue à Paris en avril 1968, André Patry soutenait que seule une crise sérieuse entre Ottawa et Québec, ou entre Ottawa et Paris, obligerait désormais le Québec à se présenter au sein d'une délégation canadienne. D’ailleurs, Patry n’accepterait qu'une délégation composée des représentants des gouvernements provinciaux qui recevraient leurs directives de leur capitale respective et parleraient seulement au nom de leur gouvernement. Le fonctionnaire affirmait que l'impasse entre Québec et Ottawa quant à la compétence en matière de politique étrangère ne se réglerait que par la négociation et qu'il était donc essentiel pour le Québec d'accumuler les précédents en faveur de la doctrine Gérin-Lajoie ${ }^{37}$. C'est pourquoi Daniel Johnson ignora les supplications de Lester Pearson pour qu'un compromis soit trouvé, et ceci, jusqu'à la veille de la réunion de Paris. Il réaffirma le droit du Québec d'y assister en vertu de la doctrine Gérin-Lajoie. Trois mois plus tard, afin d'afficher son opposition à l'interprétation fédérale de la compétence en matière de politique étrangère, Québec refusa l'invitation fédérale à se joindre à une délégation canadienne envoyée à une réunion de l'UNESCO à Paris ${ }^{38}$.

$$
\div+\div
$$

35. Claude MORIN, op. cit., p. 114 et 124.

36. BAnQ, P776, 2001-01-006, vol. 3, Dossier Personnel-Télégramme de Morin à Chapdelaine, 8 janvier 1969, Conférence des ministres de l'Éducation, Kinshasa.

37. BAnQ, E42, 1995-02-001, vol. 147, CME, Statut du Québec, Général, Note de Patry à Johnson, 9 avril 1968, Les conférences internationales en matière d'éducation; BAnQ, E42, 1995-02-001, vol. 147, CME, Statut du Québec, Général - Note de Patry à Johnson, 10 avril 1968, Fédéralisme et conférences internationales sur l'éducation.

38. Claude MORIN, op. cit., p. 136-137 ; Renée LESCOP, Le Pari québécois du général de Gaulle, Montréal, Les Éditions du Boréal Express, 1981, p. 76. 
L'intensification de la rivalité entre Québec et Ottawa au sujet de l'aide au développement en Afrique francophone est symptomatique de l'objectif visé par les deux capitales afin d'accumuler les précédents en faveur de leur position respective ${ }^{39}$. Bien que le Québec ne disposât pas des mêmes ressources qu'Ottawa, Jean Chapdelaine se rassurait du fait que l'influence fédérale en Afrique était quelque peu éphémère puisqu'Ottawa ne pouvait retirer son aide à un pays sans provoquer une réaction néfaste chez les autres ${ }^{40}$. Dans l'exportation en Afrique de sa rivalité avec Ottawa, le Québec pouvait aussi compter sur l'influence de la France, comme en témoignent les circonstances entourant la participation du Québec à la réunion des ministres francophones de l'Éducation à Kinshasa en 1969.

Chapdelaine avait sonné l'alarme et souligné que le précédent établi à Libreville ne garantissait pas sa répétition à Kinshasa et qu'il était tout à fait possible qu'Ottawa soit alors invité à la place du Québec. Le délégué général reçut des informations de ses contacts parisiens selon lesquelles le Québec, moyennant une offre d'aide, pourrait compter sur une invitation officielle. Il considéra pourtant qu'une offre de 500000 \$ pour une école ou un autre établissement constituait un prix trop élevé à payer pour une invitation distincte, ce qui allait placer le Québec dans une situation difficile au sein de la Francophonie ${ }^{41}$. Claude Morin fit valoir qu'il était essentiel que le Québec soit invité et qu'il ait sa propre délégation à la réunion : la participation du Québec à une délégation fédérale enfreignait la doctrine GérinLajoie et suggérait une renonciation à ses revendications ${ }^{42}$. Les deux fonctionnaires songèrent alors à un boycott pour protester contre l'absence d'une invitation pour le Québec et saper la capacité d'Ottawa à former une délégation légitime, mais l'intervention de Paris auprès de Kinshasa rendit cette mesure inutile : l'ambassadeur de la France à Kinshasa annonça que France tolérait une délégation canadienne à la réunion, mais qu'elle n'y assisterait pas à moins que le Québec ne reçoive une invitation distincte. Le Zaïre fut donc contraint d'adresser une invitation au Québec afin d'éviter l'échec de la conférence ${ }^{43}$.

$$
4 \div
$$

39. Au sujet de cet aspect de la rivalité, voir Robin GENDRON, op. cit., p. 87-88 et 108

40. BAnQ, P762, 1999-10-011, vol. 61, Relations internationales du Québec, Conférence de Niamey au Niger, 1969-1970 - Télégramme de Chapdelaine à Morin, 28 novembre 1968, Niamey.

41. BAnQ, E42, 1995-02-001, vol. 147, Statut du Québec: CME Conférence générale, 1968 - Lettre de Chapdelaine à Morin, 19 septembre 1968.

42. BAnQ, E42, 1995-02-001, vol. 147, Statut du Québec: CME Conférence générale, 1968 - Claude Morin, Quelques brèves réflexions sur Kinshasa, 20 décembre 1968.

43. BAnQ, P776, 2001-01-006, vol. 3, Dossier Personnel - Télégramme de Morin à Chapdelaine, 8 janvier 1969, Conférence des ministres de l'Éducation, Kinshasa; BAnQ, E42, 1995-02-001, vol. 147, 
À Québec, le Consul général de France, Pierre de Menthon, sonna la victoire de la "bataille de Kinshasa » : bien que l'effet du précédent de Libreville n'ait pas été absolu puisque le Canada avait été invité, l'appui français semblait néanmoins avoir permis au Québec de diminuer l'influence d'Ottawa au Zaïre en empêchant une victoire fédérale totale et en évitant une perte substantielle des gains québécois effectués au Gabon ${ }^{44}$. Même si la déclaration de victoire de de Menthon se révéla prématurée, puisque le Président zaïrois Joseph-Désiré Mobutu "désinvita " plus tard le Québec, soulignant qu'il fallait que la délégation québécoise fasse partie de la délégation canadienne, il ne s'agissait pas d'un revers aussi important que l'ont soutenu J.L. Granatstein et Robert Bothwell. En effet, au moment de la "désinvitation ", Ottawa et Québec réalisèrent un compromis qui permit à la délégation québécoise de s'exprimer sur ses compétences au sein de la délégation canadienne. Le ministre d'État à l'Éducation, Jean-Marie Morin, fut nommé coprésident de la délégation canadienne à Kinshasa avec le premier ministre du Nouveau-Brunswick, Louis Robichaud. En fait, il y avait pour ainsi dire deux délégations quasi-indépendantes à Kinshasa plutôt que d'une seule délégation canadienne unie ${ }^{45}$.

Pendant ce temps, Claude Morin exprimait ses réserves sur la position doublement ambiguë du Québec : d'un côté, dépendant de l'aide française et, de l'autre, en concurrence constitutionnelle avec Ottawa. Il plaida : «[n]ous sommes fortement débiteurs, un objet de politique plutôt qu'un sujet. Ce n'est pas une situation confortable, ni qui puisse continuer longtemps, ni qui le doive, sans que nous y perdions la direction de nos affaires ${ }^{46}$ ». L'implication d'Ottawa au sein de la Francophonie devenait de plus en plus importante, ce qui convainquit Morin que le moment était propice à la poursuite de négociations en vue d'établir qu'une participation distincte et autonome soit garantie à une délégation québécoise à l'intérieur

$$
+\div+\div
$$

Statut du Québec, CME Conférence générale, 1968 - Télégramme de Chapdelaine à Morin, 21 décembre 1968 ; BAnQ, P776, 2001-01-006, vol. 3, Dossier Personnel - Télégramme de Morin à Chapdelaine, 8 janvier 1969, Conférence des ministres de l'Éducation, Kinshasa.

44. MAE, vol. 213 - Lettre de de Menthon à personne anonyme, MAE, 8 janvier 1969; BAnQ, P776, 2001-01-006, vol. 3, Dossier Personnel - Télégramme de Morin à Chapdelaine, 8 janvier 1969, Conférence des ministres de l'Éducation, Kinshasa.

45. J.L. GRANATSTEIN et Robert BOTHWELl, op. cit., p. 139; Eldon BlaCK, Direct Intervention: CanadaFrance Relations, 1967-1974, Ottawa, Carleton University Press, 1997, p. 86; Claude MORIN, op. cit., p. 148-153. Jean-Guy Cardinal refusa de présider une délégation résultant de ce compromis fédéralprovincial.

46. BAnQ, P776, 2001-01-006, vol. 3, Dossier Personnel - Télégramme de Morin à Chapdelaine, 8 janvier 1969, Conférence des ministres de l'Éducation, Kinshasa. 
de la représentation canadienne ${ }^{47}$ dans toutes les conférences portant sur des sujets de compétence provinciale. Morin reconnaissait le caractère novateur de ce qu'il proposait pour le droit international, mais il croyait qu'il s'agissait de la solution la moins dommageable pour les deux parties dans la conjoncture constitutionnelle et politique de l'époque, et qu'il fallait donc convaincre Ottawa d'être assez sage pour ne pas engager le Québec dans une situation qui pourrait s'avérer néfaste pour tous.

La solution envisagée par Morin apparaissait d'autant plus pressante à la veille de la conférence de Niamey que les balises d'une institutionnalisation de la Francophonie devaient y être établies. En effet, la création d'une agence intergouvernementale pour faciliter la coopération entre les pays francophones soulevait la question du statut du Québec au sein de celle-ci et concernait directement le conflit opposant déjà Québec et Ottawa quant aux compétences canadiennes et québécoises en affaires étrangères. Selon Morin : "Tous les gains québécois internationaux réalisés jusque-là, s’il était à la rigueur impossible de les effacer entièrement, se réduiraient à bien peu de choses, à peine un accident de parcours, si jamais le gouvernement fédéral réussissait à empêcher le Québec d'accéder à l'Agence ${ }^{48}$ ".

Encore une fois, c'est une intervention française qui assura la sauvegarde des intérêts québécois : une autre menace de boycott français mena à une invitation distincte pour le Québec à Niamey. L'affaire confirmait la validité de la réflexion de Morin quant aux rapports ambigus entre Paris, Québec et Ottawa. En effet, Paris exerça, à l'insu du Québec, une pression sur le Président nigérian, Hamani Diori, pour que seul le Québec soit invité. Ottawa compta finalement au nombre des invités, mais l'intervention française poussa Ottawa et Québec à faire un compromis semblable à celui réalisé pour la réunion à Kinshasa ${ }^{49}$. La délégation québécoise à Niamey, présidée par Marcel Masse, forma un groupe distinct de la délégation canadienne. De plus, Ottawa accepta à contrecœur que la délégation canadienne s’abstienne de voter si un désaccord survenait entre les deux délégations. D'ailleurs,

$$
\stackrel{+}{+\cdots}
$$

47. On emploie l'expression " une représentation canadienne " plutôt que "délégation canadienne " parce que des acteurs québécois tels Claude Morin et Marcel Masse soutenaient que, puisque les sujets abordés étaient de compétence provinciale, Ottawa ne pouvait pas "déléguer » quelqu’un pour parler et agir en son nom. Seuls le Québec et les autres provinces possédaient cette compétence.

48. Claude MORIN, op. cit., p. 198-199.

49. BAnQ, P776, 2001-01-006, vol. 4, Niamey, 1969-1970 - Télégramme de Morin à Chapdelaine, 21 février 1969, Niamey; DAE, A-3-c, vol. 10687, 26-4-1969-NIAMEY, p. 1 - Lettre de Diori à Trudeau, 18 novembre 1968; DAE, A-3-c, vol. 10687, 26-4-1969-NIAMEY, p. 1 - Télégramme de Malone, Canadian High Commission, Lagos, au DAE, 20 novembre 1968, Francophonie Conference in Niamey; Claude MORIN, op. cit., p. 184-187. 
suivant en cela la logique de la doctrine Gérin-Lajoie, Masse affirma qu'il n'y avait pas une délégation per se à Niamey, mais plutôt une « représentation canadienne " puisque, compte tenu du fait que la plupart des activités projetées de l'ACCT étaient de compétence provinciale, seul le Québec disposait de la capacité à y être représenté ${ }^{50}$.

La conférence de Niamey aboutit à la création d'un secrétariat provisoire dont le mandat était de rédiger les statuts de l'ACCT qui seraient approuvés lors d'une réunion subséquente, encore une fois à Niamey. Dans les mois suivants, Jean Chapdelaine évoqua l'exemple du traité de Versailles dans l'histoire constitutionnelle canadienne pour illustrer l'importance des gains effectués par le Québec à Niamey I. Le délégué général soulignait à cet égard la sagesse du gouvernement britannique qui avait préféré la voie pragmatique à l'intransigeance. Selon Chapdelaine, la reconnaissance de la divisibilité de la Couronne, qui avait permis au Canada d'avoir sa propre délégation au sein de la délégation impériale britannique à la conférence de Paris, puis un siège à la Société des Nations, avait assuré le maintien d'un empire britannique au bord de l'éclatement en raison des velléités autonomistes de ses dominions. Chapdelaine était toutefois d'avis que la sagesse politique britannique de l'époque n'existait plus au Canada. Le Québec devait donc forcer la main d'Ottawa, quitte à recourir à un certain chantage pour établir un nouvel équilibre à l'intérieur de la fédération. Ottawa devait pour sa part accepter un partage des pouvoirs dont il disposait par tradition et par défaut. Chapdelaine soutenait que l'aide française constituait un grand atout à cet égard et qu'il importait de s'en servir pour négocier un modus vivendi avec le palier fédéral. Il concédait l'existence d'une alternative indépendantiste, mais croyait que la conjoncture politique du Québec rendait celle-ci peu pratique $^{51}$.

L'approche pragmatique et conservatrice dont la recommandation de Chapdelaine constitue un exemple frappant fut aussi manifeste lors du deuxième volet de la conférence de Kinshasa, tenu à Paris. Pendant les préparatifs, Claude Morin signala au Consulat général de la France à Québec que si le Québec tenait "en l'occurrence à recevoir une invitation directe, ce n'[était] pas nécessairement parce que nous nous prépar[i]ons à nous séparer

$$
+\div
$$

50. BAnQ, P776, 2001-01-006, vol. 4, Niamey, 1969-1970 - Télégramme de Morin à Chapdelaine, 21 février 1969, Niamey; Claude MORIN, op. cit., p. 154-155; Eldon BLACK, op. cit., p. 90-91. Les décisions à la conférence sont prises d'un commun accord, de sorte que cette entente demeura lettre morte.

51. BAnQ, P776, 2001-01-006, vol. 3, Dossier Personnel - Télégramme de Chapdelaine à Héroux, pour Claude Morin, 21 novembre 1969. 
du reste du pays : nous voul[i]ons simplement faire prévaloir notre conception de la constitution canadienne qui, selon nous, prévo[ya]it que l'éducation ressort[1̂]t exclusivement aux attributions des provinces ${ }^{52}$ ». Le fait que Morin intervint auprès des autorités françaises pour exprimer qu'Ottawa soit informé officiellement et assiste à la réunion de Paris est une nouvelle illustration de l'effort québécois pour accumuler les précédents tout en évitant une confrontation majeure au sujet de l'aide française. Il estimait qu'il était dans le meilleur intérêt du Québec d'éviter un éclat qui puisse provoquer la perte des gains récents, affirmant que «mieux valait profiter des circonstances encore relativement favorables pour cristalliser dans l'usage les meilleurs arrangements possibles, si bien qu'à la longue nous établirions un certain nombre de "droits acquis" $53_{\text {". }}$.

La nécessité pour le Québec d'en arriver à un modus vivendi avec le fédéral pour fixer ses gains à propos de son activité internationale et de sa participation à la Francophonie était plus grande que jamais : pendant les mois qui précédèrent Niamey II, Ottawa s'efforça d'astreindre Québec et Paris à reconnaître la primauté, sinon l'exclusivité, de la prérogative fédérale en matière de politique étrangère ${ }^{54}$. En effet, à la suite de l'affaire du Gabon, Ottawa publia Fédéralisme et conférences internationales sur l'éducation, qui affirmait l'indivisibilité de la souveraineté du Canada ${ }^{55}$. Encore une fois, les actions de la France furent cruciales dans l'évolution des événements. Réagissant à une requête québécoise, le secrétaire d'État français aux Affaires étrangères, Jean de Lipkowski, intercéda auprès du président Diori pour que seul le Québec soit admis à l'ACCT, en vertu de l'argument suivant lequel l'intérêt d'Ottawa pour l'Afrique francophone diminuerait dès que la participation distincte du Québec dans la Francophonie serait empêchée. De plus, il soutint que le fait que la population du Québec était majoritairement francophone, et que cette population représentait $80 \%$ des francophones du

$$
4+4
$$

52. Cité dans Frédéric BASTIEN, op. cit., p. 53.

53. Claude MORIN, op. cit., p. 159-161 ; Frédéric BASTIEN, op. cit., p. 54 ; Eldon BLACK, op. cit., p. 88 et 124. Ottawa citait l'exemple de Libreville pour faire valoir qu'elle avait automatiquement le droit d'assister au deuxième volet à Paris en vertu de sa participation à Kinshasa. Morin a reconnu le rôle que l'attitude du premier ministre Jean-Jacques Bertrand, plus conciliant envers le niveau fédéral, a joué dans la décision du Québec. Un compromis Québec-Ottawa de dernière heure a abouti à une délégation présidée par Jean-Marie Morin, le ministre d'État à l'Éducation, accompagné de représentants du Québec, des autres provinces et de conseillers fédéraux.

54. J.L. GRANATSTEIN and Robert BOTHWELl, op. cit., p. 148.

55. DAE, A-3-c, vol. 10046, 20-FR-1-2, p. 13 - Télégramme du DAE à l'ambassade du Canada, Paris, 3 April 1968, CDA-FRA Relations: Conversation with French Ambassador; Mitchell Sharp, Fédéralisme et conférences internationales sur l'éducation, Gouvernement du Canada, 1968; Mitchell SHARP, Which Reminds Me...: A Memoir, Toronto, University of Toronto Press, 1994, p. 188; Eldon BLACK, op. cit., p. 35. 
Canada, plaidait en faveur d'une inclusion à part entière du Québec ${ }^{56}$. Deux mois plus tard, à la fin de novembre 1969, l'ambassadeur français au Niger communiqua à Diori les préoccupations de Paris : "éviter toute procédure qui paraitrait une conception unitaire [de l'ACCT] empêchant le Québec de s'exprimer $^{57}$ ». Le président français, Georges Pompidou, qualifiait l'invitation du Québec "d'indispensable». En février 1970, il permit à son secrétaire général aux Affaires africaines et malgaches, le très influent Jacques Foccart, d'exercer des pressions sur Diori ${ }^{58}$. Les démarches de Foccart furent suivies de celles du secrétaire d'État aux Affaires étrangères, de Lipkowski, qui informa les capitales africaines du fait que le Québec ne participerait pas à Niamey II sans une invitation directe. À propos de la participation fédérale, il nota qu' «il serait dommage qu'un pays anglephone [sic] ait ainsi réussi à imposer sa volonté à un ensemble francophone ${ }^{59}$ ».

Malgré toutes ces interventions françaises, le Québec ne reçut pas d'invitation à Niamey II. En partie en raison des pressions fédérales, Diori se contenta d'informer le premier ministre Jean-Jacques Bertrand de la tenue de la réunion, soulignant ainsi indirectement son accord avec l'interprétation fédérale de la compétence en politique étrangère. Selon Morin, "le Québec risquait de subir un recul politique de taille " puisque placé devant une institutionnalisation possible de la Francophonie sans qu'une participation distincte ne lui soit ménagée ${ }^{60}$.

Dans les semaines qui précédèrent la conférence, alors que Québec et Ottawa étaient dans l'impasse à propos des modalités d'une participation du Québec, des discussions eurent lieu à Paris sur un report possible de Niamey II ou un boycott français. Au-delà de ses intérêts spécifiques, Paris se montrait réticent à traiter avec une délégation canadienne «à vocation anglophone " et des représentants des États de l'Afrique francophone «influencés largement " par Ottawa ${ }^{61}$. En dépit des indications initiales que Chapdelaine reçut à l'effet qu'un boycott québécois serait suivi d'une

$$
\div+\div
$$

56. MAE, vol. 232 - Note pour le Secrétaire d'État, 16 septembre 1969, Entretien de M. de Lipkowski avec M. Hamani Diori.

57. Claude MORIN, op. cit., p. 207.

58. Jacques FOCCART, Journal de l'Elysée, tome 3. Dans les bottes du général. (1969-1971), Fayard/Jeune Afrique, 1999, p. 234-235; Frédéric BASTIEN, op. cit., p. 61

59. DAE, A-3-c, vol. 8647, 20-1-2-FR, p. 26 - Télégramme de l'ambassade du Canada, Paris, au DAE, 20 février 1970, Niamey II ; DAE, A-3-c, vol. 10691, 26-4-1970-NIAMEY, p. 2 - Télégramme de de Goumois, Délégation canadienne, Niamey, à Bissonnette, DAE, 12 mars 1970, FrancophonieNiamey II.

60. Claude MORIN, op. cit., p. 205 et 210.

61. Archives nationales de France, 5AG 2/1039 - Note de Raimond à Jobert, 6 mars 1970, Conférence de Niamey. 
demande de report de la part de Paris, les intérêts supérieurs de la France en Afrique prévalurent et la réunion eut lieu. Pompidou lui-même interprétait la méthode pragmatique de Québec comme la preuve d'une attitude attentiste. À la fin de janvier 1970, il décrivait le Québec comme «un partenaire très mollasson ", divisé intérieurement ${ }^{62}$. Le Québec se trouvait donc tout à coup dans une position difficile à un moment crucial dans sa concurrence avec Ottawa.

Devant ce revers et la possibilité d'une défaite vis-à-vis du pouvoir fédéral, Chapdelaine suggéra à Claude Morin qu’un échec de la création de l'ACCT ne serait pas catastrophique. En dépit du fait que Paris cherchait une formule susceptible à la fois de rallier les Africains et de permettre une participation éventuelle du Québec à la Francophonie, Morin demeurait très pessimiste en raison de la fermeté du gouvernement fédéral et ce qu'il décrivait comme le "retournement français". La seule arme dont disposait le Québec, selon Morin, était la menace d'un boycott ${ }^{63}$. Bien que le premier ministre Bertrand se montrait plus conciliant avec Ottawa que son prédécesseur, il n'était pas disposé à sacrifier les gains du Québec relativement à sa capacité internationale et à sa participation dans la Francophonie. De toute façon, l'aurait-il voulu que sa position politique l'en aurait empêché. Par conséquent, Bertrand fit une allusion détournée à un boycott québécois dans sa réponse à l'avertissement fédéral selon lequel le Canada retirerait son appui à l'ACCT si la France ou un autre pays tentait de saper la position fédérale quant à sa compétence en affaires étrangères. Bertrand prétendit qu'il y avait "un seuil de dignité» que le Québec ne pouvait pas franchir. Il affirma que la présence et les activités du Québec au sein de la Francophonie devaient être clairement identifiées, que le Québec devait pouvoir parler en son nom et s'impliquer dans les domaines qui relevaient de sa compétence grâce à une participation directe aux travaux de l'ACCT ${ }^{64}$.

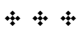

62. Jacques FOCCART, Journal de l'Elysée, tome 3. Dans les bottes du général. (1969-1971), Paris, Fayard/Jeune Afrique, 1999, p. 237-241 et 253-254. Au début, Pompidou a donné son appui à un boycott québécois, car «nous faisons la francophonie pour le Québec, et ce serait complètement ridicule de la faire avec le Canada sans le Québec». Pourtant, en même temps, le président avait le souci que l'appui français soit mesuré, craignant que le Québec s'en serve pour frapper Ottawa et que Paris en fasse les frais.

63. BAnQ, P776, 2001-01-006, vol. 4, Niamey, 1969-1970 - Note au dossier, Jean Chapdelaine, 5 mars 1970, Niamey; BAnQ, P776, 2001-01-006, vol. 4, Niamey, 1969-1970-Télégramme de Morin à Chapdelaine, 9 mars 1970, Niamey.

64. DAE, A-3-c, vol. 10691, 26-4-1970-NIAMEY, p. 1 - Lettre de Trudeau à Bertrand, 6 mars 1970 ; BAnQ, P762, 1999-10-011, vol. 62, Relations internationales du Québec, Documents portant sur la participation du Québec à l'ACCT, 1970-1971 - Télégramme de Bertrand à Trudeau, 10 mars 1970 ; BAnQ, P776, 2001-01-006, vol. 4, Niamey, 1969-1970 - Lettre de Morin à Chapdelaine, 13 avril 1970. 
Face à un probable boycott québécois, Ottawa, ne voulant pas porter le blâme de l'échec possible de Niamey II et du projet de l'ACCT, accepta la plupart des demandes québécoises à propos du choix de ses délégués et concéda au chef de la délégation québécoise le droit de parler au nom du Québec à propos des sujets de juridiction provinciale. Malgré ce succès, aucune garantie ne découla de cette concession quant à l'utilisation éventuelle qu'Ottawa pourrait faire de la charte de l'ACCT pour faire reculer les gains récents du Québec ${ }^{65}$.

Toutefois, ce fut la proposition française pour l'admission à l'ACCT de "gouvernements participants" des États non souverains, qui disposaient à la fois d'une compétence constitutionnelle sur les sujets traités par l'agence et de l'approbation de leur autorité centrale, qui l'emporta. La plupart des délégations à Niamey II s’y rallièrent. Ottawa finit par emboîter le pas à contrecœur après l'échec de sa contre-proposition qui stipulait que la proposition française ne s'appliquerait pas aux États fédéraux qui étaient euxmêmes des membres de l'ACCT, ce qui aurait eu pour effet d'empêcher une participation distincte pour le Québec. Julien Chouinard, secrétaire au Cabinet québécois et chef de la délégation québécoise à la conférence, signa la charte de l'ACCT pour le Québec à titre de membre de la délégation canadienne ${ }^{66}$.

Les responsables québécois furent très heureux du résultat : le Québec devint effectivement membre d'une organisation internationale et le fond de la doctrine Gérin-Lajoie - selon laquelle le Québec avait une capacité internationale dans les domaines qui relevaient de sa compétence constitutionnelle - fut confirmé et institutionnalisé. Jean Chapdelaine fut de l'avis que le Canada s'était engagé, en voulant contenir le Québec, dans une opération qui dépassait ses moyens et ses capacités et qu'il avait par conséquent été remis à sa place. Le Québec, quant à lui, récupéra une marge de manœuvre pour régler avec Ottawa la question de son admission formelle à l'ACCT en tant que "gouvernement participant». De plus, Chapdelaine espérait que le résultat établirait un précédent pour une consultation et une coopération du même genre à l'UNESCO et à l'OCDE. Morin se fit l'écho de l'analyse de Chapdelaine et, utilisant une analogie empruntée au poker,

$$
\div+
$$

65. J.L. GRANATSTEIN and Robert BOTHWEll, op. cit., p. 149 ; Claude MORIN, op. cit., p. 218. 66. DAE, A-3-c, vol. 10691, 26-4-1970-NIAMEY, p. 2 - Télégramme de Pelletier, Délégation canadienne, Niamey, au DAE, 18 mars 1970, Conf.-Niamey II : DAE, A-3-c, vol. 8647, 20-1-2-FR, p. 27, Mémoire au Cabinet, 19 mars 1970, Niamey II - Attitude envers la France; Frédéric BASTIEN, $o p$. cit., p. 66-68 ; J.L. GRANATSTEIN and Robert BOTHWELL, op. cit., p. 149-152. 
affirma qu'avec l'aide française, le Québec avait battu un «flush » avec une paire de deux ${ }^{67}$.

À la fin de Niamey II, le Québec se trouva dans une position vis-àvis d'Ottawa qui rappelle celle qu'occupait le Canada au sein de l'Empire britannique après le traité de Versailles et avant la déclaration Balfour de 1926 : il parvint à affirmer une capacité internationale et se trouva sur le point d'être admis comme membre d'une organisation internationale, bien que ses relations avec le pouvoir central restassent à régler. Cet aboutissement fut dû en grande partie aux adroites stratégies des fonctionnaires québécois qui profitèrent de l'aide française dans leur concurrence constitutionnelle avec Ottawa. Dans un rapport produit par le ministère français des affaires étrangères, Niamey II fut présenté comme le plus récent effort de Paris pour remettre entre les «mains des Québécois un certain nombre d'atouts politiques inespérés "; c'était "à eux désormais d'en jouer ${ }^{68}$ ". Paris avait doublé Ottawa et ses tentatives pour bloquer une participation distincte du Québec à l'ACCT. À l'époque de la conférence de paix à Paris et dans les années qui avaient suivi, des circonstances particulières avaient obligé Londres à accepter la présence des dominions sur la scène mondiale ; maintenant, c'était Ottawa qui était forcé par l'action gaulliste d'accepter l'action internationale du Québec. Depuis cette époque, le Québec a pu utiliser ces atouts pour s'assurer, à plusieurs reprises, une place à la table internationale. L'accord passé avec le gouvernement Mulroney quant à la participation de Québec aux Sommets de la Francophonie et, plus récemment, celui signé entre les gouvernements Charest et Harper à propos de la place du Québec à l'UNESCO, ont pu être conclus en vertu des négociations de 1971.

Laide française n'explique toutefois qu'en partie ce résultat. Bien sûr, comme des études antérieures sur l'action internationale du Québec l'ont déjà démontré, l'aide parisienne fut tout à fait cruciale pour l'obtention d'une participation distincte du Québec au sein de la Francophonie et, plus largement, pour l'affirmation de sa capacité internationale dans les années soixante. Mais le désir d'un rapprochement avec le monde francophone et le besoin, dans un contexte de mondialisation croissante, de se projeter au-delà des rivages canadiens afin notamment de se protéger des empiétements fédéraux, ne devraient pas être sous-estimés lorsqu'on examine les motivations et

$$
\div+\div
$$

67. BAnQ, P776, 2001-01-006, vol. 4, Niamey, 1969-1970 - Conférence de Niamey II, 16-20 mars 1970, rapport de Jean Chapdelaine, 31 mars 1970 ; BAnQ, P776, 2001-01-006, vol. 4, Niamey, 1969 1970 - Lettre de Morin à Chapdelaine, 13 avril 1970.

68. MAE, vol. 218 - Note pour Monsieur le Ministre, 3 octobre 1970, Impressions canadiennes. 
les actions du Québec à cette époque. Pourtant, de manière consciente ou non, les moyens retenus par les personnes ayant joué un rôle de premier plan dans l'action internationale du Québec étaient en accord avec le patrimoine britannique, partie intégrante de la culture politique du Québec et caractéristique centrale de son évolution historique. Envisagé sous cet angle, l'effort québécois pour participer à la Francophonie apparaît plus qu'une fin en soi; c'était aussi un moyen pour arriver à d'autres fins. En effet, dans leur lutte pour accumuler des précédents, les représentants québécois s'inspiraient de l'expérience canadienne vieille d'un demi-siècle et espéraient répéter le succès rencontré par Ottawa à l'époque où le Canada progressait lentement vers la souveraineté internationale. Qu'ils aient été à Québec ou à Ottawa, qu'ils aient cherché à accumuler ou, à l'inverse, à limiter le nombre de précédents, les participants à ce jeu politique et constitutionnel partageaient les mêmes références nourries par le constitutionnalisme canado-britannique ${ }^{69}$. Il est ironique de constater que pendant les années soixante, au moment même où de Gaulle, les membres du lobby du Québec à Paris et les fonctionnaires français célébraient les retrouvailles et les relations particulières entre les "Français de France» et les «Français du Québec», ceux-ci étaient engagés, sans en être pleinement conscients, dans ce qui peut être décrit comme une opération québécoise « very British».

$$
4+4
$$

69. À cet égard, voir la discussion du concept de canadianité dans Jocelyn LÉTOURNEAU, op. cit., chapitre 3 . 